Paidéia, 2006, 16(35), 377-384

\title{
FATORES DE RISCO NA ADOLESCÊNCIA: DISCUTINDO DADOS DO DF ${ }^{1}$
}

\author{
Kátia Tarouquella Brasil \\ Paola Biasoli Alves ${ }^{2}$ \\ Deise Matos do Amparo \\ Kellen Cristine Frajorge \\ Universidade Católica de Brasília
}

\begin{abstract}
Resumo: Este estudo teve por objetivo investigar e discutir situações de risco psicossocial em adolescentes do Distrito Federal tomando por base a Teoria dos Sistemas Ecológicos. Foram aplicados 852 questionários em adolescentes de escolas públicas do DF, localizadas em regiões previamente selecionadas e com indicadores de risco psicossocial. Os resultados mostram que a violência, as tentativas de suicídio, a presença de drogas e a sexualidade são fatores de risco para os jovens. Contudo, estes adolescentes têm desenvolvido estratégias efetivas para a promoção de sua saúde, como o uso de preservativos nas relações sexuais, a convivência familiar cotidiana, a expectativa de apoio de vizinhos e líderes comunitários. Percebe-se que esta pode ser uma realidade específica do DF e salienta-se a necessidade de construção de Políticas Públicas que beneficiem as questões de prevenção à violência intrafamiliar, e também a obtenção de sustentabilidade econômica destes jovens.
\end{abstract}

Palavras-chave: Adolescência; Risco Psicossocial; Violência; Estratégias Protetivas; Políticas Públicas.

\section{RISK FACTORS IN ADOLESCENCE: DATA FROM DF/BRAZIL}

Abstract: This study had as objectives to investigate and to discuss the risk situations for adolescents from the Distrito Federal, Brazil, based on the Ecological Theory Approach. 852 questionnaires were applied in adolescents from the public schools of the DF, localized in some regions previously identificated as in risk situation. The data show that the violence, the suicide attempt, the presence of drugs and the sexuality are indicators of risk. Nevertheless, the same data show that these adolescents are capable of developing protective strategies to the promotion of their health such as condom use in their sexual relationships, family daily contact and support expectation from the neighborhood. On observe that hese data can be specific from the DF but it is necessary to create and to apply Public Politcs related to the domestic violence prevention and to the economical support to this young people.

Key words: Adolescence; Psycho-Social Risk; Violence; Protective Strategies; Public Politcs.

\section{Introdução}

O processo da adolescência aparece como tema de investigação de várias disciplinas nas Ciências Humanas, e este interesse partilhado demarca também uma dificuldade comum, a saber, um mesmo espírito de condescendência epistemológica que considera a ausência da generalidade do conceito de adolescência (Reis \& Zioni, 1993). A Psicologia do Desenvolvimento, com ênfase na perspectiva interacionista, busca contextualizar, no

${ }^{1}$ Recebido em 05/10/2006 e aceito para publicação em 04/01/2007. ${ }^{2}$ Endereço para correspondência: Paola Biasoli Alves, Rua 30 Sul, lote 08, Edifício Bouganville, apto. 407, CEP: 71929-360, Taguatinga - DF, E-mail: paolabia@yahoo.com âmbito histórico-social, o desenvolvimento do adolescente enquanto indivíduo integral, que não é criança, nem adulto. A adolescência caracteriza-se como processo intermediário e distinto destes e traz questões que lhe são particulares, pois além das mudanças nos aspectos orgânicos, os adolescentes compõem um grupo sócio-cultural específico, com um modo único de se interar com seus pares e também com o mundo (Traverso-Yepez, \& Pinheiro, 2002). Segundo a Organização Mundial da Saúde (OMS), a adolescência corresponde a um período em que: a) o indivíduo passa do ponto do aparecimento inicial dos caracteres sexuais secundários para a maturidade sexual; b) os seus processos psicológicos 


\section{Kátia Tarouquella Brasil}

e as formas de identificação evoluem da fase infantil para a adulta; c) a transição do estado de dependência econômica total passa a outro de relativa independência. Entretanto, a OMS entende que não é possível impor limites específicos à adolescência, e que este termo corresponde a uma classificação social que varia tanto em sua composição como nas implicações. Assim, a cronologia entre 10 e 20 anos não delimita a adolescência, mas, permite as reflexões sobre as dimensões bio-psico-sociais que interagem na construção da identidade e da ação adolescente no mundo contemporâneo, sendo ponto inicial para a convergência das definições do processo de adolescer. $\mathrm{Na}$ atualidade, somam-se às questões cronológicas os aspectos hormonais e fisiológicos, as conquistas e funções cognitivas, os parâmetros sócio-afetivos, a construção dos recursos intrapsíquicos e as macro-dimensões históricas, sociais e morais (Reis \& Zioni, 1993).

Diante da multiplicidade característica deste processo de desenvolvimento, construiu-se uma leitura cuidadosa em relação às experiências particulares vividas na adolescência e destaca-se na atualidade a de situação de risco psicossocial, compreendida como toda e qualquer vivência em que o jovem experimenta o estresse de forma significativa em sua vida, seja por questões de características pessoais, seja pelas sócio-ambientais (Amparo, Alves \& Cárdenas, 2004; Morais \& Koller, 2004). Desta forma, tem-se a investigação e análise dos fatores de risco como importantes aspectos que inscrevem o adolescente em uma problemática particular. Os fatores de risco individuais encontram-se relacionados às características como gênero, problemas genéticos, carência de habilidades sociais, intelectuais e características psicológicas limitadas; e os de riscos ambientais, como a violência, ausência ou fragilidade de suporte social e afetivo e o baixo nível sócio econômico (Paludo \& Koller, 2005). Deste modo, o termo comportamento de risco refere-se às ações que se caracterizam como ameaças ao desenvolvimento saudável que o adolescente pode alcançar superando situações aversivas, que segundo a Teoria dos Sistemas Ecológicos não são isoladas, mas integradas às vivências dos processos proximais, ou seja, nas interações estabelecidas com pessoas, objetos e símbolos através de atividades cotidianas, de desempenho de papéis e da internalização de significados, tanto no seu microssistema, quanto na integração deste com o meso e macro sistemas (Martins \& Szymanski, 2004; Morais \& Koller, 2004; Yunes, Miranda \& Cuello, 2004). Sabe-se que a qualidade das interações vivenciadas ao longo de todo o ciclo vital é influente na exposição ao risco e, também, na promoção da resiliência, definida como a retomada de processos saudáveis de desenvolvimento quando há exposição ao risco (Amparo, Alves \& Cárdenas, 2004; Martins \& Szymanski, 2004).

Assim, de acordo com a Teoria dos Sistemas Ecológicos, os processos proximais mantidos pelos adolescentes têm características pertencentes a sua cultura e são instrumentos centrais na formação de sua identidade, contendo a dinâmica de risco e também de proteção para o desenvolvimento (Amparo, Alves \& Cárdenas, 2004; Martins \& Szymanski, 2004).

$\mathrm{Na}$ atualidade a concepção de risco na adolescência assume uma configuração singular, na medida em que se relaciona à exposição, violência, drogas e precocidade das experiências sexuais. Estes fatores apresentam-se como de risco para o desenvolvimento quando o adolescente os encontra no cotidiano de sua comunidade, ou seja, quando permeiam seus processos proximais; e também por sua dimensão macrossistêmica, em que questões políticas, culturais e ideológicas sustentam padrões desfavoráveis à saúde do desenvolvimento.

Nesse contexto, a Teoria dos Sistemas Ecológicos contribui para a compreensão das diversidades que marcam a população de jovens desta pesquisa, bem como, a interação deles e seu ambiente, uma vez que esse modelo tem como objeto de estudo o homem social inserido em um contexto natural. Assim, não se pensam os fatores de risco de forma isolada, independente e fragmentada, pois a exposição ao perigo que os potencializa ocorre de diversas formas e em vários contextos. O ambiente ecológico de desenvolvimento humano não se limita a um único e imediato; deve ser concebido como estruturas concêntricas interrelacionadas que afetam o desenvolvimento do indivíduo. E os diversos tipos fazem emergir diferentes padrões de com- 
portamentos, sentimentos e pensamentos, ampliando ou estagnando os processos daqueles que estão inseridos em todos os graus dos sistemas e contextos (Bronfenbrenner, 1993, 1996; Morais \& Koller, 2004).

A concepção de desenvolvimento humano da Teoria dos Sistemas Ecológicos encontra-se dividida em quatro níveis dinâmicos interrelacionados, $o$ tempo, a pessoa o processo e o contexto. Nessa perspectiva, ela é contextual e se constitui a partir de processos proximais através dos quais as particularidades da pessoa e do ambiente interagem produzindo constância e mudança nas características pessoais no decorrer da vida (Bronfenbrenner, 1993, 1995).

O Processo é definido pelo modo de relação entre as características da pessoa e o ambiente e ele se caracteriza em proximais. O segundo componente do modelo é a Pessoa, com suas características biopsicológicas e as que se constroem através da interação com o ambiente. O Contexto possui quatro níveis ambientais: o microssistema, em que se identificam as interações face-a-face, o mesossistema, que é composto pela relação entre os micro, o exossistema, sendo aquele em que a pessoa focalizada não participa efetivamente, mas cujos eventos influenciam na sua vida e o macrossistema, que se constitui pelos valores, crenças culturais, sociais e pelas ideologias. Por último, as mudanças e continuidades que ocorrem ao longo do ciclo de vida são analisadas pelo Tempo (Morais \& Koller, 2004).

Dentro destes parâmetros, a violência é entendida como intervenção física ou simbólica, realizada de forma impositiva e claramente amparada em relações de desequilíbrio de poder, de um indivíduo ou grupo contra outro (Pegoraro, 2002). As estatísticas oficiais apontam no sentido de uma tendência mundial, desde os anos 50 do século XX, para o crescimento dos crimes e da violência social e interpessoal. No Brasil, o alvo preferencial de morte por homicídio compreende adolescentes e jovens adultos masculinos, em especial procedentes das chamadas classes populares urbanas (Adorno, 2002). Percebe-se aqui a profunda integração entre as vivências cotidianas dos processos proximais (experiências de abandono, de agressão, de instabilidade afetiva) e os aspectos macrossistêmicos como a urbanização sem planejamento, a ausência de políticas públicas efetivas nas áreas de saúde e educação, emprego, entre outros fatores (Adorno, 2002).

Ao enunciar a droga como um fator de risco é preciso tratar esta questão na versão da atualidade, entendida como aquilo que orienta os adolescentes na busca de um elemento mágico que os distancia dos laços sociais e do reconhecimento de um sofrimento inerente à existência humana. É difícil separar e isolar os efeitos que o grupo de pares tem sobre os adolescentes e se o que está em questão não é a droga em si, e sim, a relação que o indivíduo estabelece com ela, que influencia e é influenciada fortemente pelo universo de interações (Birman, 1999; Levisky, 1995). Uma leitura mais acurada da experiência da adolescência aponta-a como um período em que os conflitos psicossociais, a necessidade de integração social, a busca da autoestima e de independência familiar colocam o sujeito em uma posição de vulnerabilidade, que pode legitimar sua busca por um alívio rápido dentro de uma sociedade em que o tempo é também um produto a ser consumido (Birman, 1999). Por outro lado, as substâncias psicoativas encontram sua expressão no eixo e macrossistemas, através do tráfico como forma de subsistência e enriquecimento econômico, da desigualdade e da injustiça social trazendo estigmas e preconceitos com relação a comunidades economicamente desfavorecidas e que convivem com o comércio ilegal de drogas. Percebe-se, assim, mais uma vez a dinâmica entre a formação da identidade adolescente e o convívio com as dimensões históricas e sócio-culturais (Amparo, Alves \& Cárdenas, 2004; Santana, Doninelli, Frosi \& Koller, 2004; Yunes, Miranda \& Cuello, 2004).

Outro aspecto de risco refere-se à precocidade do início da vida sexual, tendo em vista os elementos relacionados à fecundidade, com os quais os adolescentes devem lidar como, por exemplo, o uso de contraceptivos e a gravidez, bem como a prevenção das DSTs/AIDS. A precocidade da vida sexual e reprodutiva no Brasil encontra-se refletida no aumento da Taxa Específica de Fecundidade (TEF) do grupo de idade de 15 a 19 anos, em contraposição à queda observada em todos os outros 
grupos (ABEP-Associação Brasileira de Estudos Populacionais, 2006). A partir deste quadro também é preciso considerar a dinâmica pessoal de cada adolescente, assim como as crenças e valores quanto à sexualidade, que são construídos nas sociedades ocidentais e que, com o caminhar histórico, possibilitam inúmeras recombinações entre faixa etária indicada para possibilidade reprodutiva e qualidade de alimentação, orientação sexual e experimentação, modalidades de relacionamentos afetivos e fidelidade conjugal, entre tantas outras.

Assim, reconhecer que o desenvolvimento adolescente inscreve-se na particularidade cultural construída a partir da modernidade, como também que a vivência do estresse econômico e afetivo interage com seu estar no mundo, são parâmetros da realização da pesquisa aqui apresentada.

A cidade de Brasília caracteriza-se por particularidades que trazem reflexos para toda uma geração que nela nasce e cresce. Ela ocupa um espaço idealmente planejado com funções prédefinidas e com a pretensão inicial de se constituir como uma cidade sem barreiras sociais. Entretanto, o Estado não teve controle sobre o fluxo migratório e, com a garantia dos direitos individuais, a população de Brasília não respeitou o planejamento idealizado por seus fundadores; as pessoas que vieram para a cidade se engajaram em um projeto nacional divulgado pelos meios de comunicação da época de sua construção, mas também continuam a vir, vislumbrando ultrapassar a barreira sócio-econômica de que se sentem prisioneiros (Nunes, 1999).

Neste trabalho pretendeu-se analisar especificamente os comportamentos e fatores de risco a que estão expostos os adolescentes do Distrito Federal. Os dados, levantados através de questionário elaborado para uso em contexto nacional, permitiram identificar e abordar aspectos referentes aos seguintes fatores e comportamentos de risco: violência (intrafamiliar e na comunidade), drogas, sexualidade e tentativas de suicídio.

\footnotetext{
3 "Pesquisa sobre a Juventude Brasileira" - questionário elaborado em 2004, utilizado atualmente e ainda não publicado.
}

\section{Método}

\section{Participantes}

Os participantes da pesquisa compõem um grupo de 852 indivíduos jovens, cursando o ensino médio em escolas públicas das regiões administrativas do Distrito Federal - Planaltina, Brazlândia, Paranoá e Samambaia, com idade entre 13 e 27 anos, sendo que $68,5 \%$ da amostra concentra-se entre 15 e 17 anos. Cerca de $57,4 \%$ dos participantes são do sexo feminino, $41,5 \%$ do sexo masculino e $1,1 \%$ não se identificou. A renda salarial familiar dos participantes encontra-se na faixa de 300 a 600 reais $(44,8 \%)$ e 600 e 1000 reais $(22,4 \%)$, sendo que 19,0 $\%$ relatam renda superior a 1200 reais. Também, $52,4 \%$ dos participantes têm pais que são migrantes do interior de outros estados do Brasil, sendo os filhos da primeira e da segunda geração de famílias que vieram para a Capital Federal em busca do sonho de uma cidade nova, com oportunidades principalmente de se distanciarem da miséria econômica deixada em suas cidades de origem. A proximidade do poder contribui para que as populações desfavorecidas, que almejam uma possibilidade concreta de acesso a melhores condições de vida, migrem constantemente para Brasília.

\section{Instrumento}

Para a coleta de dados, utilizou-se o questionário construído por Koller, Cerqueira-Santos, Morais \& Ribeiro ${ }^{3}$ (2004) que consta de 109 questões de múltipla escolha. Este instrumento está sendo aplicado em outras capitais brasileiras e focaliza o perfil de adolescentes em situação de risco psicossocial, investigando questões sobre resiliência e vulnerabilidade. Buscou-se, com esse instrumento, um levantamento dos fatores de risco e proteção que afetam a vida de jovens brasileiros, além de aspectos sobre sua caracterização bio-sócio-demográfica e dados sobre as temáticas de educação, saúde, trabalho, violência, lazer, religiosidade, rede de apoio social, humor, otimismo, auto-estima, e auto-eficácia.

\section{Procedimentos}

Para a escolha das escolas utilizou-se os seguintes critérios: localização (áreas com indicadores de pobreza tais como condições precárias de moradia), idade dos jovens (de 13 a 23 anos) e 
nível sócio-econômico dos adolescentes (médio e baixo $)^{4}$. Para coleta de dados, em um primeiro contato, os pesquisadores visitaram as escolas e apresentaram informações sobre o projeto, visando a inserção dos pesquisadores na escola de forma participativa e integrada. Em seguida, os questionários foram aplicados em grupo. Todos os alunos foram voluntários e assinaram o Termo de Consentimento Livre e Esclarecido.

\section{Resultados e Discussão}

Os fatores de risco interferentes na complexidade do desenvolvimento dos adolescentes, discutidos nessa pesquisa por meio da análise dos indicadores referentes aos comportamentos de risco, apresentam-se integrados às dimensões: violência (atos de violência e confronto com a lei), violência intrafamiliar e na comunidade, exposição e contato com drogas, incidência de suicídio, além da temática da prevenção de DST/ AIDS.

Os dados mostram que $22,3 \%$ dos adolescentes têm alguém da família que está ou já esteve preso e apenas 3,2\% deles esteve internado em instituições. Nos dados sobre violência estão inseridas as respostas às questões que apresentam indicadores de violência social, física, sexual e emocional no âmbito doméstico ou social amplo, caracterizando a temática nas diferentes dimensões do contexto ecológico (micro, meso e macrossistemas). Assim, pode-se perceber que os processos criminais e infracionais fazem parte do mesossistema dos adolescentes, contudo, eles se mantêm distantes destas experiências enquanto fator de risco individual. Neste sentido, pode-se inferir que a violência apresenta-se, para este grupo, enquanto processo simbólico e interativo na perspectiva macrossistêmica, ou seja, é possível trazer sofrimento e angústia a partir de sua dimensão cultural, na qual são vividas experiências relacionadas ao preconceito e aos estereótipos sócio-econômicos (Santana, Doninelli, Frosi \& Koller (2004); Yunes, Miranda \& Cuello, 2004).

\footnotetext{
${ }^{4}$ O Distrito Federal apresenta um dos maiores Índices de Desenvolvimento Humano do Brasil (IDHs), assim, a seleção da região para coleta dos dados foi realizada considerando as áreas do entorno com menores IDHs, através dos seguintes critérios: renda familiar de dois salários mínimos e meio, índice de analfabetismo elevado (12,3\%) e condições precárias de moradia $(9,6 \%$ de barracos).
}

Na dimensão mesossistêmica, de acordo com os processos proximais estabelecidos dentro da própria comunidade, os dados mostram que $85,4 \%$ dos jovens sentem-se, entre inseguros e mais ou menos seguros quanto a sua cotidianidade nestes locais. Estes dados relacionam-se com os índices de tráfico, assaltos e roubos na comunidade, que são elevados, sendo de $46,1 \%$ e $66,7 \%$ respectivamente. Também, há o relato de batidas policiais em $31,1 \%$ das respostas, aspecto que evidência a situação de insegurança vivida nas comunidades. Pode-se assinalar que a qualidade da rede de apoio sócioafetivo comunitária, na avaliação destes jovens sobre a segurança e as ações policiais, encontra-se fragilizada e com possibilidade de criar situações de distanciamento de suas origens familiares. Esta análise encontra ressonância no fato de que $75,7 \%$ dos jovens pesquisados nunca ou às vezes percebese como pertencente a sua comunidade. Porém, ao serem identificadas as expectativas quanto a ajuda de vizinhos e lideranças comunitárias, os jovens assinalam níveis médios e elevados de expectativa em $60,0 \%$ e $64,9 \%$ dos casos, respectivamente.

Ao entender a dinâmica da construção de identidade na interação entre as dimensões da pessoa, do processo, do tempo e do contexto, a temática do suicídio apresenta-se como um indicador significativo na análise dos fatores de risco vividos pelos jovens, uma vez que $10,2 \%$ deles já tentou suicídio. Destes $57,1 \%$ fizeram uma tentativa, $28,6 \%$ duas, $11,7 \%$ tentaram três vezes e para $2,6 \%$ há mais de três tentativas. Os motivos que justificaram este comportamento segundo os participantes da pesquisa foram: problemas familiares $(74 \%)$, falta de sentido para viver $(59,7 \%)$, desilusão amorosa $(23,9 \%)$, dificuldades financeiras $(13,4 \%)$ e uso de drogas $(1,5$ $\%)$. Assim, como um tema relacionado à violência, com características de impacto subjetivo fortemente marcadas, o tema do suicídio apresenta dados inerentes às dimensões pessoais destes jovens, sendo que os motivos elencados priorizam as interações no microssistema familiar e, também, a constituição dos recursos psíquicos imprescindíveis para a sustentação bio-psico-social de qualquer indivíduo e, na perspectiva da situação de risco, fatores de proteção potenciais para a vivência resiliente. 


\section{Kátia Tarouquella Brasil}

Vale destacar que para os adolescentes participantes da pesquisa, o grupo familiar é uma referência importante, consistindo-se em um significativo eixo de apoio e rede afetiva contextual, uma vez que o desenvolvimento humano ocorre por processos progressivos de interações duradouras, como é o caso das relações familiares (Martins \& Szymanski, 2004). Os adolescentes apontam que pais e mães co-habitam os lares com eles, apresentando respectivamente um percentual de $69,6 \%$ para os pais e $91,7 \%$ para as mães. Assim, mesmo na situação prevista de risco em função de questões econômicas e sociais, as habilidades, competências e recursos desenvolvidos na convivência familiar saudável aparecem, para este grupo de jovens, como um indicador de constituição de identidade dentro de parâmetros protetivos. Porém, quando há a análise em relação à exposição ao risco no grupo intrafamiliar, $15,3 \%$ dos jovens vivem situações de alcoolismo na família, 5\% apontam a existência de violência nela, $2 \%$ indicam ter tido relações sexuais forçadas. Estes dados evidenciam a realidade de convivência com episódios desfavoráveis ao desenvolvimento, mesmo que de uma parcela da amostra, o que direciona a discussão de Políticas Públicas fortemente sistematizadas em torno dos temas de prevenção da violência intrafamiliar e da promoção de aspectos saudáveis da socialização das novas gerações, sendo que os indicadores da existência de conflitos não podem ser considerados exclusivamente como indicadores de violência, mas se referem ao estabelecimento de limites (BiasoliAlves \& Dessen, 2001).

Os principais resultados encontrados, no que se refere às questões sobre sexualidade indicam que $60,0 \%$ dos respondentes ainda não teve sua primeira relação sexual; porém dos 38,4 \% com experiência sexual, a menor idade observada foi de 10 anos e a mais frequiente 15 anos. Dos que tiveram relação sexual, $86,7 \%$ afirmam que nunca engravidaram, $68,1 \%$ sempre utilizam métodos anticoncepcionais e 93,5\% sempre se preveniram de DSTs, gravidez e AIDS pelo método da camisinha. Este dado permite, novamente, a reflexão sobre a integração entre risco e proteção na vida destes jovens, pois apesar das adversidades cotidianas, eles conseguem desenvolver estratégias de cuidado e proteção quanto aos riscos da vivência sexual desprotegida. Considerando que a incidência de doenças sexualmente transmissíveis vem aumentando entre adolescentes, e que os fatores responsáveis são a idade de início das relações sexuais, o aumento do uso de parceiros e a ausência de preservativos (Chequer, 1998), nota-se que os adolescentes da pesquisa possuem um perfil diferenciado para a efetivação de estratégias protetivas, uma vez que são capazes de diminuir a incidência de risco, recorrendo ao uso da camisinha.

Em relação às drogas lícitas como uso de álcool, $46,9 \%$ dos respondentes diz que já experimentaram e 53,1 \% não relatam experiência com bebidas alcoólicas. A maior parte dos adolescentes (74\%) nunca experimentaram cigarro. Quanto ao uso de drogas ilícitas, os percentuais de uso são considerados baixos. Os adolescentes indicam que experimentaram maconha $(5,3 \%)$, haxixe $(0,8 \%)$, cola $(1,2 \%)$, lança perfume $(3,0 \%)$ loló $(1,0 \%)$, cocaína $(2,6 \%)$, crack $(1,4 \%)$. Pode-se perceber que a questão das drogas no cotidiano destes jovens vincula-se mais fortemente ao uso de álcool e que a experimentação de outras substâncias psicoativas é restrita. Estes dados corroboram com a perspectiva do risco enquanto possibilidade macrossistêmica na vida destes jovens e que existem oportunidades relevantes para a elaboração de diferentes propostas em Políticas Públicas que promovam e implementem a sua qualidade de vida (Silva \& Santos, 2001).

O conjunto de dados apresentados, na análise dinâmica proposta pela Teoria dos Sistemas Ecológicos, evidencia a integração entre as possibilidades de risco e proteção na vida dos indivíduos e a necessidade de pesquisas contextuais para que Políticas Públicas possam ser criadas e efetivadas dentro das mais diferentes comunidades jovens no Brasil (Morais \& Koller, 2004). As questões presentes no instrumento aplicado, referentes aos riscos na vida dos jovens pesquisados, encontram-se integradas aos aspectos contemporâneos de dimensões sociais e psíquicas e, em suas respostas, foi possível identificar a criação de estratégias protetivas, mesmo estando presentes os indicadores de risco. Percebe-se que esta pode ser uma realidade inerente às características particulares do DF, 
contudo, salienta-se a adolescência enquanto processo de construção de identidade, pautada na consciência de si e do outro, e na busca de estruturas que favoreçam o desenvolvimento saudável. Assim, se esta é uma busca do indivíduo, cabe aos órgãos competentes a estruturação e implementação de ações voltadas para a promoção do bem-estar da sociedade em geral, o que significa estabelecer o movimento de integração entre pessoa, processo, tempo e contexto como base de reflexão e, principalmente, de prática efetiva na direção da saúde destes jovens. A pesquisa realizada mostra a força e o impacto das vivências e expectativas individuais interagindo com as situações sociais e, desta forma, evidencia as dimensões contextuais e pessoais como integrantes e constituintes de um conjunto, favorecendo o pensamento e a ação, enquanto interação e construção de processos de desenvolvimento.

\section{Referências}

ABEP-Associação Brasileira de Estudos Populacionais.(2006). <http://www.abep.org.br/ usuario/GerenciaNavegacao.php?cademo id $=019$ \&nivel $=2>$. Acesso 25 de Setembro2006.

Adorno, S. (2002). Exclusão socioeconômica e violência urbana. Sociologias, 4(8), 84-135.

Amparo, D. M., Alves, P. B., \& Cárdenas, C. J. (2004). Pertencimento e identidade em adolescentes em situação de risco de Brasília. Revista Brasileira de Crescimento e Desenvolvimento Humano, 14(1), 1-13.

Biasoli-Alves, Z. M. M., \& Dessen, M. A. (2001). O estudo da família como base para a promoção da tolerância. Em Z. M. M. Biasoli-Alves \& R. Fischmann (Orgs.), Crianças e adolescentesConstruindo uma cultura da tolerância.(pp.183193). São Paulo: EDUSP/UNESCO.

Birman, J. (1999). Mal-Estar na atualidade: a psicanálise e as novas formas de subjetivação. Rio de Janeiro: Civilização Brasileira.

Bronfenbrenner, U. (1993). The ecology of cognitive development: research models and fugitive findings. In R. Wosniak \& D. Fischer (Eds.), Development in context, acting and Thinking in speci-fic environments. (pp. 3-44). Hillsdale (NJ): Erlbaum.
Bronfenbrenner, U. (1995). Developmental ecology through space and time: A future perspective. In P. M. Moen, G. H. Eldre \& K. Lüscher, (Eds.), Examining lives in context. (pp. 619-648). Washington (DC): American Psychological Association.

Bronfenbrenner, U. (1996). A ecologia do desenvolvimento humano: Experimentos naturais e planejados. Porto Alegre: Artes Médicas. (Trabalho originalmente publicado em 1979).

Chequer, P. (1998). A AIDS no Brasil: Perfil epidemiológico e ações. A Folha Médica, 117(1), 3-5.

Levisky, D. (1995). Adolescência: Reflexões psicanalíticas. Porto Alegre: Artes Médicas.

Martins, E., \& Szymanski, H. (2004). A abordagem ecológica de Urie Bronfenbrenner em Estudos com famílias. Estudos e Pesquisa em Psicologia, 1(4), 66-77.

Morais, N. A., \& Koller, S. H. (2004). Abordagem Ecológica do Desenvolvimento Humano, Psicologia Positiva e Resiliência: Ênfase na saúde. Em S. H. Koller (Org.), A ecologia do desenvolvimento humano: Pesquisa e intervenções no Brasil. (pp.91-108). São Paulo: Casa do Psicólogo.

Nunes, B. F. (1999). O Espaço Social de Brasília. O Descontrole Planejado. Série Sociológica, 174. Brasília-DF.

Paludo, S. S., \& Koller, S. H. (2005). Resiliência na rua: um estudo de caso. Psicologia: Teoria e Pesquisa, 2 (21), 187-195.

Pegoraro, J. S. (2002). Notes on youth bearing juvenile violence within post-industrial societies. Sociologias, 8 (4), 276-317.

Reis, A. O. A., \& Zioni, F. (1993). O lugar do feminino na construção do conceito de adolescência. Revista de Saúde Pública, 6 (27), 472-477.

Santana, J. P., Doninelli, T. M., Frosi, R. V., \& Koller, S. H. (2004). As instituições de atendimento a jovens em situação de rua segundo a Abordagem Ecológica do Desenvolvimento Humano. Em S. H. Koller (Org.), A ecologia do desenvolvimento humano: Pesquisa e intervenções no Brasil. (pp. 189-196). São Paulo: Casa do Psicólogo. 
Silva, R. C., \& Santos, M. A. (2001). A intolerância frente a questão das drogas: Algumas reflexões. Em Z. M. M. Biasoli-Alves \& R. Fischmann (Orgs.), Crianças e adolescentesConstruindo uma cultura da tolerância. (pp.131148). São Paulo: EDUSP/UNESCO.

Traverso-Yepez, M. A., \& Pinheiro, V. S. (2002). Adolescência, saúde e contexto social: esclarecendo práticas. Psicologia e Sociedade, 2(14), 133-147.

Yunes M. A. M., Miranda, A. T., \& Cuello, S. E. S. (2004). Um olhar ecológico para os riscos e as oportunidades de desenvolvimento de crianças e adolescentes institucionalizados. Em S. H. Koller (Org.), A ecologia do desenvolvimento humano: Pesquisa e intervenções no Brasil. (pp.197-218). São Paulo: Casa do Psicólogo. 